\title{
Die grosse Rede des Timaios - ein Beispiel wahrer Rhetorik?
}

\author{
Lucius Hartmann
}

\begin{abstract}
The great cosmological speech of Timaios points in comparison to similar written texts of the 4 th century to a number of peculiarities, and even inside the corpus Platonicum the text appears singularly. These remarkable features can be explained by the consequent application of the philosophical rhetoric - the soul conducted by words -, conceived by Platon mainly in the dialogs Gorgias and Phaedrus. The most important criteria are knowledge (especially of ideas), a good structure with the definition of central terms, the application of a scientific psychology and a critical attitude to the value of written texts. Timaios, as an exceptionally gifted astronomer, a politically successful orator and a true philosopher meets these requirements nearly perfect.
\end{abstract}

\section{Keywords}

Plato - Phaedrus - Gorgias - Timaeus - rhetoric - psychology

Liest man Platons Dialog Timaios und vergleicht die darin enthaltene grosse Rede des Timaios mit den Reden anderer Autoren des 5. oder 4. Jahrhunderts vor Christus, kann man zahlreiche Eigentümlichkeiten erkennen. ${ }^{1}$

So geht der Rede erstens ein dialogisches Einleitungsgespräch voraus, in welchem der Redner ebenso wie die drei Zuhörer - im Vergleich zu zeitgenössischen Reden ein geradezu verschwindend kleines Publikum - charakterisiert werden und der Vortrag der Rede motiviert wird. Und in der Fiktion des

1 Kurzfassung der Dissertation, die im Februar 2016 an der Universität Zürich angenommen und 2017 unter dem gleichen Titel publiziert wurde, vgl. Hartmann Die grosse Rede des Timaios - ein Beispiel wahrer Rhetorik. Angesichts der Kürze des Beitrags wird weitgehend auf Begründungen verzichtet, ebenso auf eine intensive Auseinandersetzung mit der Forschung. Als Übersetzungen wurden verwendet: Susemihl (zeno.org) für den Timaios und Georgii (zeno.org) teilweise für den Phaidros (markiert mit *).

(C) LUCIUS HARTMANN, 2021 | DOI:10.1163/9789004437081_003

This is an open access chapter distributed under the terms of the CC BY-NC-ND 4.olicense fartmann - 9789004437081 
Timaios wird die Rede kurz nach Beginn sogleich wieder unterbrochen, um den Zuhörern die Möglichkeit zu geben, ihre Zustimmung zu äussern (Tim. 29d).

Zweitens beginnt Timaios seine Ausführungen sehr atypisch mit einem Anruf der Götter (27c), den man sonst höchstens in der Dichtung noch findet. ${ }^{2}$

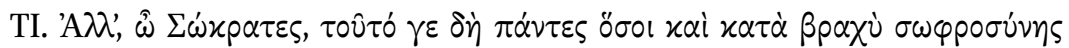

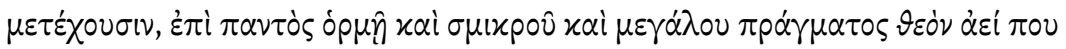

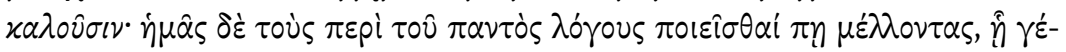

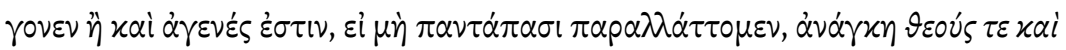

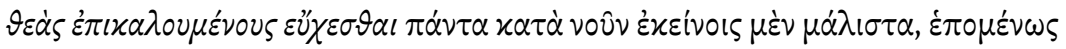

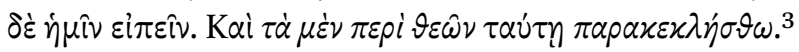

(Timaios:) Traun, lieber Sokrates, tun doch das wohl alle, die auch nur ein wenig Überlegung besitzen: rufen doch sie alle wohl beim Beginne eines jeden Unternehmens, mag es nun geringfügig oder bedeutend sein, stets einen Gott an. Und wir, die wir gar über das All zu sprechen im Begriffe sind, nämlich inwiefern es entstanden ist oder aber unentstanden von Ewigkeit war, müßten ja ganz und gar den Verstand verloren haben, wenn wir nicht die Götter und Göttinnen anrufen und von ihnen erflehen wollten, daß es uns gelingen möge, das Ganze vor allem nach ihrem Sinne, sodann aber auch in Übereinstimmung mit uns selber darzulegen. Und so mögen denn die Götter eben hierum angerufen sein.

Tim. 27c f.

Drittens wird die Rede vom Autor gleichzeitig als mythos und als logos bezeichnet und oszilliert damit zwischen dieser Antithese der griechischen "Aufklärung“. ${ }^{4}$ Die Grenzen der menschlichen Erkenntnis und die Überlegenheit der Götter werden von Timaios in seiner Rede explizit thematisiert (29c f.), und dadurch scheint er seine Ausführungen grundsätzlich zu relativieren.

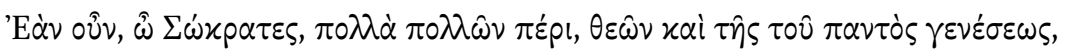

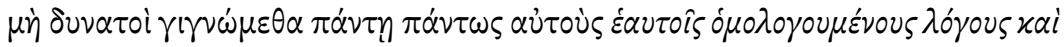

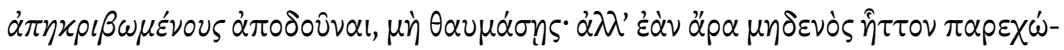

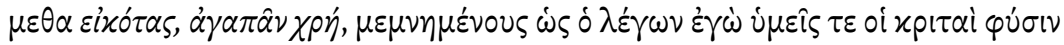

2 Inhaltlich nahe ist z.B. Empedokles' naturphilosophisches Gedicht Peri physeōs (DK ${ }_{31} \mathrm{~B}_{3}$ ).

3 Alle griechischen Texte sind dem TLG entnommen.

4 Mythen werden auch den „modernen“ Sophisten zugeschrieben: Prodikos (Xenophon, Mem. II 1.21-34) und Protagoras (Platon, Prot. $320 \mathrm{ff}$.$) .$ 


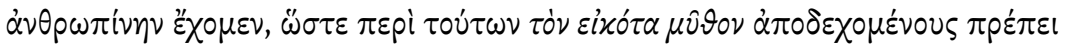

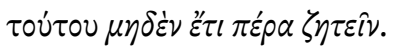

Wenn ich daher, mein Sokrates, trotzdem daß schon viele vieles über die Götter und die Entstehung des Alls erörtert haben, nicht vermögen sollte, eine nach allen Seiten und in allen Stücken mit sich selber übereinstimmende und ebenso der Sache genau entsprechende Darstellung zu geben, so wundere dich nicht; sondern wenn ich nur eine solche liefere, die um nichts minder als die irgend eines anderen wahrscheinlich ist, so müßt ihr schon zufrieden sein und bedenken, daß wir alle, ich, der Darsteller, und ihr, die Beurteiler, von nur menschlicher Natur sind, so daß es sich bei diesen Gegenständen für uns ziemt, uns damit zu begnügen, wenn die Dichtung nur die Wahrscheinlichkeit für sich hat, und wir nichts darüber hinaus verlangen dürfen.

Tim. 29c f.

Viertens wirkt die Darstellung von komplexen mathematischen und naturwissenschaftlichen Zusammenhängen und Erkenntnissen in einer derart langen Rede fürs 4. Jahrhundert bereits anachronistisch, da sie ihre Parallelen eigentlich nur in geschriebenen Texten (z.B. der Medizin) findet.

Doch nicht nur neben den zeitgenössischen Reden scheint der Timaios auffallend, sondern auch innerhalb von Platons Werk. Wie kommt es, dass sich Sokrates (zwar nur als Zuhörer) mit einer naturphilosophisch geprägten Erklärung unserer Welt beschäftigt, einem Thema, von dem er sich gemäss eigener Aussage im Phaidon nach seinen schlechten Erfahrungen mit Anaxagoras (Phd. 97b ff.) längst gelöst hat und von dem er der Apologie zufolge gar nichts versteht (Apol. 19c)?

Weiter vertritt Timaios ganz eindeutig das Prinzip des eikos (Tim. 29c f.), ein von Platon überaus kritisch eingeschätztes Beweisverfahren der sophistischen Rhetorik.

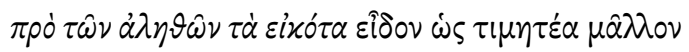

(Sokrates:) < Gorgias und Teisias> erkannten, dass das Wahrscheinliche mehr als das Wahre geschätzt werden müsse.

Phdr. $267 \mathrm{a} 6 \mathrm{f}$.

Schliesslich scheint auch die Länge der Rede nicht unproblematisch: Im Gorgias droht Sokrates seinem Gesprächspartner Polos, er werde sich aus dem Gespräch zurückziehen, wenn Polos weiterhin der makrologia fröne (Gorg. 461d f.), und ebenso will Sokrates den Dialog mit Protagoras abrupt 
beenden, wenn dieser seine Antworten nicht kürzer fasst (Prot. 334c ff.). Unter diesen Voraussetzungen wirkt es seltsam inkonsequent, dass Timaios' viel längere Rede Sokrates nicht im Geringsten zu stören scheint.

Umgekehrt präsentiert Platon - hauptsächlich in seinem Dialog Phaidros - eine Reihe von Kriterien für eine wahre, philosophische Rhetorik als Gegenstück zur traditionellen, sophistischen Rhetorik. ${ }^{5}$ Es stellt sich daher die Frage, ob sich unter diesen Prämissen die Eigentümlichkeiten des Timaios besser verstehen lassen und ob der Timaios allenfalls sogar als ein Beispiel dieser wahren Rhetorik betrachtet werden kann.

\section{Forschungsstand}

Auffälligerweise im Vergleich zu den Reden im Phaidros, im Symposion, im Menexenos und in der Apologie fehlen bislang Versuche fast vollständig, auch die grosse Rede des Timaios auf die Erfüllung der Kriterien der wahren Rhetorik hin zu untersuchen. ${ }^{6}$

Nach Hadot ist die Rede des Timaios ein „échantillon de cette rhétorique philosophique“, indem sie die Seele im All situiert und somit ein meteorologisches Thema, wie es im Phaidros gefordert werde, enthält, und er erklärt den eikōs logos mit den Vorgaben der Schriftkritik. ${ }^{7}$ Auch wenn beide Argumente kaum zutreffend sind, bleibt seine Schlussfolgerung dennoch richtig.

Nach Mesch ist im Timaios ebenfalls die wahre Rhetorik erfordert (und umgesetzt), da das behandelte Thema (Abbilder von Ideen und nicht Ideen selbst) im besten Fall diese Art von Rhetorik ermöglicht, weil die eigentlich höher stehende Dialektik „an eine Grenze in der dialektischen Thematisierbarkeit von Gegenständen stößt“ und damit nicht verwendet werden könne. Er erklärt ausserdem die ungewöhnliche Länge und die Bildhaftigkeit der Rede als „Sonderfall einer gegenstandsadäquaten Rhetorik". 8 Die Frage, ob diese Rhetorik auch adressatenadäquat sei, wird von ihm jedoch nur in Ansätzen beantwortet.

5 Unter dem Begriff "sophistische Rhetorik“ ist im Folgenden die gesamte nichtplatonische Rhetorik zusammengefasst (also nicht nur die Ansichten von Protagoras und Gorgias, sondern auch von Korax, Teisias, usw.).

6 Vgl. e.g. Colloud-Streit, Fünf platonische Mythen im Verhältnis zu ihren Textumfeldern, zum Phaidros, Thompson, „The Symposion: a neglected Source for Plato's Ideas on Rhetoric“, zum Symposion, Eucken, „Die Doppeldeutigkeit des platonischen Menexenos“, zum Menexenos, Colaiaco, Socrates against Athens, zur Apologie. Kritisch sind beispielsweise Werner, „Rhetoric and philosophy in Plato's Phaedrus", zum Phaidros und Guthrie, A History of Greek Philosophy III-V, zum Menexenos.

7 Hadot, „Physique et poésie dans le Timée de Platon“, 127.

8 Mesch, „Die Bildlichkeit der platonischen Kosmologie“, 203. 
Racionero sieht in der Rede des Timaios ebenfalls ein „exercise of legitimate rhetoric", wobei er in seiner Begründung ausschliesslich vom Kriterium der Wahrheit und ihrer (durch den Gegenstand der Rede bedingten) nur teilweise möglichen Kommunikation ausgeht. ${ }^{9}$

Sowohl Johansen als auch Ashbaugh zeigen aufgrund des Vergleichs einer Rede mit einem Lebewesen aus dem Phaidros (Phdr. 264c), dass Timaios' Rede diese Vorgabe an die Struktur weitgehend erfüllt. ${ }^{10}$ Brague nimmt die PhaidrosStelle sogar zum Anlass (aus meiner Sicht wenig überzeugend), die einzelnen Teile der Rede mit menschlichen Körperteilen zu vergleichen. ${ }^{11}$

In ihrem nur sehr marginal der Rhetorik gewidmeten Aufsatz sprechen auch Lampert/Planeaux (leider ohne Verweis auf den Phaidros) davon: "Timaeus-Critias is an example of that alliance of philosophy and rhetoric, the theory of which is presented in the Republic."12 Gleiches gilt auch für Nevsky, welcher den Timaios an die Politeia anschliessen lässt und ihn für „un échantillon de la bonne manière de parler « de la nature de l'univers »" hält..13

Die vorhandene Forschung bescheinigt der Rede des Timaios also die Erfüllung einzelner Kriterien der wahren Rhetorik, doch basieren diese Resultate teilweise auf falschen Annahmen oder wenig überzeugenden Übertragungen und widerspiegeln nur einen Teil der im Phaidros oder anderswo formulierten Forderungen, so dass sich bloss ein unvollständiges Bild ergibt. Ziel meiner Untersuchung ist es, diese lückenhaften Resultate durch einen möglichst systematischen und umfassenden Ansatz zu verifizieren und zu vervollständigen.

\section{Kriterien der wahren Rhetorik}

Auf der Basis hauptsächlich des Phaidros, der sich von allen Dialogen am intensivsten mit den technischen Einzelheiten der Rhetorik auseinandersetzt, sollen im Folgenden die wichtigsten Kriterien der wahren Rhetorik dargestellt werden. ${ }^{14}$

9 Racionero, „Logos, myth and probable discourse in Plato's Timaeus“, 58.

10 Johansen, Plato's Natural Philosophy: A Study of the Timaeus-Critias, Ashbaugh, Plato's Theory of Explanation, vgl. unten Anm. 32.

11 Brague, „The Body of the Speech“. So kann u.a. die Identifikation des Kopfs der Rede mit ihrem Anfang kaum richtig sein, vgl. Tim. 69 bi.

12 Lampert und Planeaux, "Who's Who in Plato's Timaeus-Critias and Why“, 121.

13 Nevsky, Voir le monde comme une image, 310.

14 Auch der Gorgias enthält neben seiner Kritik an der sophistischen Rhetorik Ansätze einer wahren Rhetorik (Gorg. 5o3a ff.), die sich nicht von derjenigen des Phaidros unterscheidet. Auf das Primat des Inhalts vor der Form weisen auch das Symposion (198b ff.), die Apologie (17a ff.) und der Menexenos (234c f.) hin. 
Die Grundidee von Sokrates' Rhetorikentwurf im Phaidros besteht darin, dass die Rhetorik (als rhētorikē techne ${ }^{-15}$ ) die Bedingungen einer technē erfüllen muss, ${ }^{16}$ d.h.

a) sie muss über Kenntnis ihres Gegenstands verfügen $(\rightarrow$ alētheia statt eikos),

b) sie muss ihre Effekte wissenschaftlich erklären können ( $\rightarrow$ Psychologie),

c) sie muss auf das Beste ausgerichtet sein ( $\rightarrow$ Ethik),

d) sie muss lehrbar sein ( $\rightarrow$ Schriftkritik).

\subsection{Kenntnis der Wahrheit (Fachwissen und Ideenwissen)}

Der Redner muss grundsätzlich die Wahrheit dessen kennen, worüber er spricht. In der Regel ist dabei auch ethisches Wissen (für die Ausrichtung auf das Gute ohnehin) notwendig, also automatisch ein Ideenwissen. ${ }^{17}$ So fragt Sokrates rhetorisch:

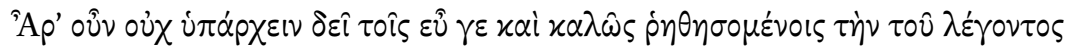

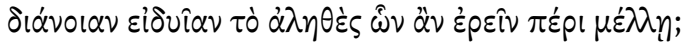

(Sokrates:) Aber muss nicht zumindest für das, was gut und schön gesagt werden soll, der Verstand des Sprechers die Wahrheit von dem wissen, worüber er sprechen will?*

Phdr. 259e

\subsection{Methode zur Erkenntnis der Wahrheit: Dialektik}

Die Erkenntnis der Wahrheit erfolgt über die Methode der Dialektik. Diese lässt sich hauptsächlich durch zwei Verfahren beschreiben:

1) Hypothesisverfahren (Phd. 10oa ff., Rep. 51ob ff., Men. 86e ff.): Man geht von einer Hypothese aus und entwickelt daraus die Konsequenzen (nach unten), dann geht man über die Hypothese hinaus und sucht sich eine weitere, übergeordnete Hypothese, bis man zur anypothetos archē

15 Zum Begriff rhētorikē vgl. Schiappa, „Did Plato coin Rhêtorikê?“, und (zurecht kritisch) Pernot, La rhétorique dans l'antiquité, $38 \mathrm{ff}$. Die Rhetorik wird im Gorgias in deutlichem

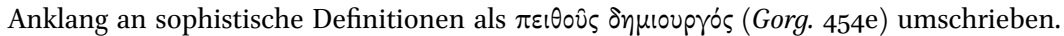
Im Phaidros ist sie als $\psi v \chi \alpha \gamma \omega \gamma i \alpha \delta \dot{\alpha} \lambda{ }^{\prime} \gamma \omega \nu$ (261a) definiert, wodurch die zentrale Rolle der Psychologie unterstrichen wird.

16 Vgl. Balansard, Technè dans les Dialogues de Platon, Brickhouse und Smith, Plato's Socrates, Heinimann, „Eine vorplatonische Theorie der $\tau \dot{\varepsilon} \chi v \eta “$.

17 Anders Heitsch, Platon, Phaidros, Übersetzung und Kommentar, vgl. aber Phdr. $260 a 1 \mathrm{ff}$. ( $\tau \dot{\alpha}$

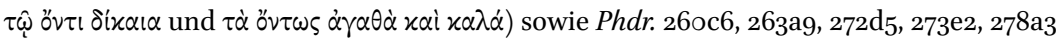
f.; die Psychologie ist ohne Ideenwissen undenkbar, ebenso die wahre Rhetorik ohne Philosophie (Phdr. 261a4 f.). 
(Rep. 510b7) gelangt; je nachdem muss die ursprüngliche Hypothese angepasst oder ganz aufgegeben werden, wenn sie zu Widersprüchen führt. ${ }^{18}$

2) Dihairesisverfahren, wie es im Phaidros explizit beschrieben ist, um Genus und Spezies eines Begriffs umfassend verstehen zu können:19

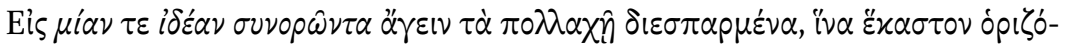

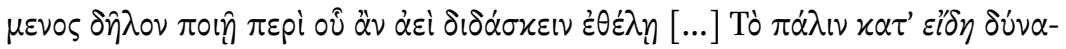

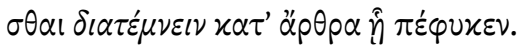

(Sokrates:) Das überall Verstreute durch den Gesamtblick in ein Genus zurückzuführen, damit man jedes, worüber man jeweils lehren möchte, klar macht, indem man es definiert [...] Umgekehrt in der Lage zu sein, es in Spezies zu unterteilen gemäss der natürlichen Gliederung.

Phdr. 265d f.

Das dialektische Verfahren (als Denkprozess) muss in der Rede selbst nicht abgebildet werden, sondern geht dieser üblicherweise voran, wie es auch aus Sokrates' Reden im Phaidros klar wird.

\subsection{Struktur}

Nach Sokrates sind die Erkenntnisse der sophistischen Rhetorik auch für die

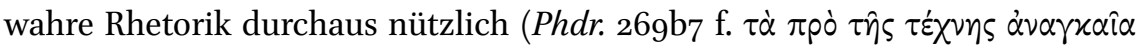
$\mu \alpha \theta \dot{\eta} \mu \alpha \tau \alpha)$. Dazu gehören insbesondere die Vorschriften zur Strukturierung von Reden, und seine eigenen Reden im Phaidros zeichnen sich in diesem Bereich ganz ausgesprochen aus. Er sieht hauptsächlich drei Kriterien: a) die Rede als Lebewesen, b) ihre Logik und c) die Notwendigkeit des Definierens.

\section{$3 \cdot 3 \cdot 1$}

\section{Lebewesen (Vollständigkeit, Wohlproportioniertheit)}

Erstens vergleicht er eine Rede mit einem Lebewesen, wobei es ihm dabei um ihre Vollständigkeit und Wohlproportioniertheit geht.

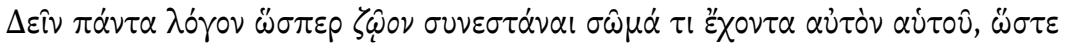

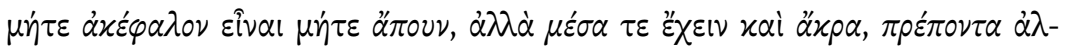

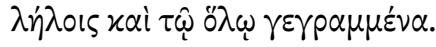

18 Vgl. u.a. Byrd, „Dialectic and Plato's Method of Hypothesis“.

19 Vgl. u.a. Sayre, Metaphysics and Method in Plato's Statesman, und Stenzel, Studien zur Entwicklung der platonischen Dialektik von Sokrates zu Aristoteles. 
(Sokrates:) Es ist notwendig, jede Rede wie ein Lebewesen aufzubauen, welches einen eigenen Körper hat, so dass es weder kopf-noch fusslos ist, sondern mittlere und äussere Teile hat, die in der Gestaltung zueinander und zum Ganzen passen.

Phdr. 264c

3.3.2 „Logik“ (Argumentation, sinnvolle Gedankenfolge)

Zweitens sollte eine Rede auch „logisch“ aufgebaut sein, d.h. eine sinnvolle, argumentativ nachvollziehbare Gedankenfolge aufweisen. ${ }^{20}$ Lysias' Rede im Phaidros hat nach Sokrates gerade dieses Kriterium nicht erfüllt:

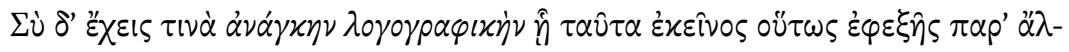

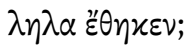

(Sokrates:) Hast du aber einen ,logographischen Zwang', nach dem jener $<$ Lysias > diese <ersten Zeilen der Rede> so in dieser Reihenfolge angeordnet hat?

Phdr. 265b

\subsubsection{Definition}

Drittenserhältjede RedeihreinhaltlicheStimmigkeit undWiderspruchsfreiheit, wenn zu Beginn die zentralen (und umstrittenen) Begriffe definiert werden. Dies wird von Sokrates in beiden Reden (im Gegensatz zu Lysias) umgesetzt, wobei die Forderung selbst in der ersten Rede zunächst auch theoretisch reflektiert wird.

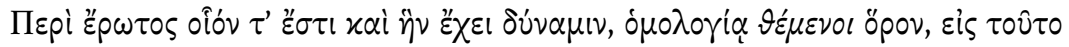

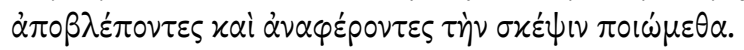

(Sokrates:) Indem wir in Übereinstimmung über den Eros festlegen, wie er ist und welche Kraft er hat, indem wir jeweils darauf blicken und uns darauf beziehen, wollen wir die Untersuchung führen.

Phdr. 237c

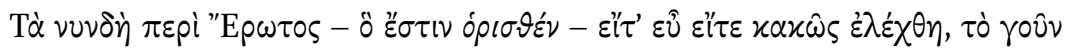

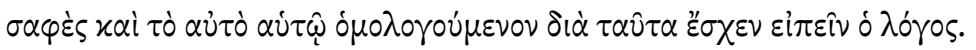

20 Heitsch, Platon, Phaidros, übersetzt mit „Kompositionsprinzip“. 
(Sokrates:) In Bezug auf das, was soeben über Eros - wie er definiert worden ist - sei es gut, sei es schlecht gesagt worden ist, konnte die Rede deswegen <wegen der Definition> wenigstens das Klare und mit sich selbst Übereinstimmende sagen.

Phdr. 265d

\subsubsection{Länge}

Im Phaidros äussert sich Sokrates nur am Rand zur Länge einer Rede (Phdr. 267b, 269a, 272a). Immerhin zeigen seine eigenen Beispiele, dass eine Rede nicht zwingend sehr kurz sein muss. Die oben erwähnten Vorwürfe an seine Gesprächspartner im Gorgias und im Protagoras sind daher unbedingt in ihrem Kontext zu lesen: Nicht lange Reden an sich sind problematisch, sondern lange Reden in einem dialektischen Gespräch mit dem Zweck, durch die Länge von dem mangelnden Inhalt abzulenken und das Gespräch zu verunmöglichen. Die Länge der Rede ist, wie dies aus dem Gorgias selbst unmissverständlich hervorgeht, abhängig vom Inhalt und insbesondere auch vom Adressaten. So muss Sokrates gegenüber Polos, den er kurz vorher gebeten hat, sich ebenfalls wie Gorgias möglichst kurz zu halten, auch einmal zu einer längeren Antwort greifen, um seine Sicht verständlich zu machen.

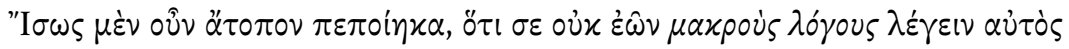

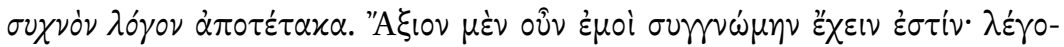

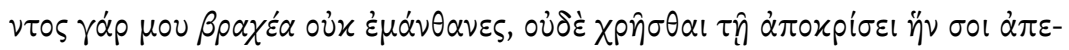
xp

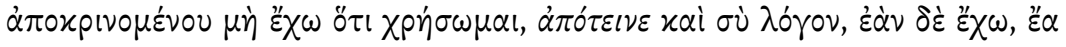
$\mu \varepsilon \chi \rho \hat{\eta} \sigma \theta \alpha$ l.

(Sokrates:) Vielleicht bin ich nun zwar unpassend vorgegangen, weil ich dich keine langen Reden halten liess, aber selbst meine Rede verlängert habe. Es ist nun freilich angemessen, mir Verständnis entgegenzubringen: denn als ich mich kurz hielt, hast du mich nicht verstanden, und $d u$ warst nicht in der Lage, mit der Antwort, die ich dir gegeben habe, etwas anzufangen, sondern du benötigtest noch eine zusätzliche Ausführung. Wenn nun freilich auch ich mit deiner Antwort nichts anzufangen weiss, verlängere auch du deine Rede, andernfalls lass es bleiben.

Gorg. 465 e f.

\subsection{Adressatenbezogenheit (Psychologie)}

Die sophistische Rhetorik sah ihre Wirkung insbesondere in der Erzeugung von pathe, und ihren Vertretern wurden teilweise magische Fähigkeiten 
zugeschrieben.$^{21}$ Sokrates anerkennt diese Leistung, aber er fordert eine profunde Kenntnis der Seele des Adressaten, um in Analogie zur Medizin eine kunstgemässe Rhetorik zu ermöglichen.

Der Redner muss demnach die Seelen der Adressaten mit einem dialektischen Verfahren (Phdr. 27oc ff.) typologisieren und ebenso die verschiedenen Reden. ${ }^{22}$ Anschliessend kann er die passenden Redetypen den Seelentypen zuordnen und ihre Effekte wissenschaftlich erklären (Phdr. 271b3 ff. $v \varphi^{\prime}$ oil $\omega \nu$

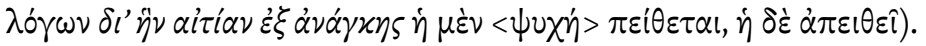

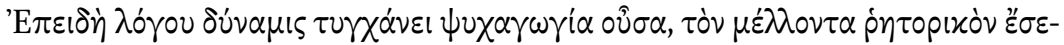

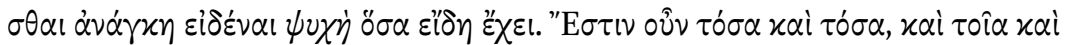

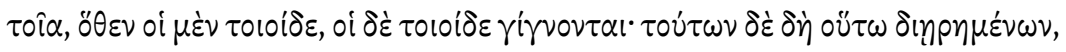

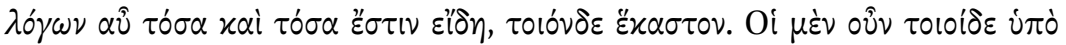

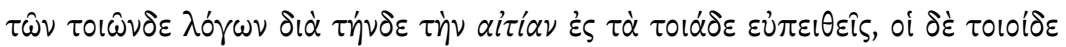

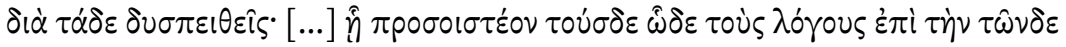

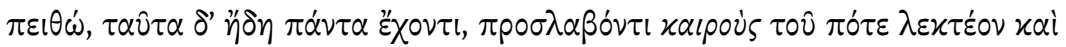

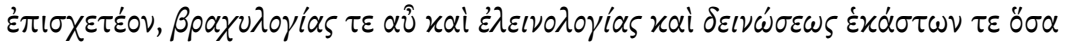

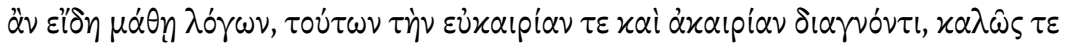

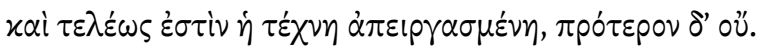

(Sokrates:) Da die Kraft der Rede eine Seelenleitung ist, so muß derjenige, der ein Redner werden will, notwendig wissen, wie viele Arten die Seele hat. Deren gibt es also so und so viele und so und so beschaffene, daher auch die Menschen einige so, andere so beschaffen sind. Nachdem aber nun dieses eingeteilt worden ist, gibt es andererseits auch so und so viele Arten von Reden, und jede so oder so beschaffen. Die so beschaffenen Menschen sind nun durch die so beschaffenen Reden aus der so beschaffenen Ursache zu den so beschaffenen Zwecken leicht zu bereden, - die so beschaffenen aber sind aus diesen Gründen schwer zu bereden. [...] < jene Natur nämlich>, bei welcher gerade diese Reden auf diese Art zur Überzeugung über diese Gegenstände angewendet werden müssen, - wenn er also dieses alles

21 Vgl. Phdr. 267c f. (zu Thrasymachos), Gorgias, Hel. 8 sowie Platon, Ion; zur Magie Menex. 235a2 und Prot. 328d4. Zur Wirkung auf Sokrates vgl. Menex. 235a ff., Symp. 198b f., Phdr. 234d, Apol. 17a. Umgekehrt wirkte auch Sokrates selbst in gleicher Weise auf seine Zuhörer, vgl. Symp. 215 $\mathrm{c} 1$ und Men. $80 \mathrm{oa} \mathrm{ff}$.

22 In Phdr. 277c spricht er von „einfachen“ (haplous, schlicht, „wissenschaftlich“, z.B. der Unsterblichkeitsbeweis Phdr. 245c ff.) bzw. „bunten“ (poikilous, ausgeschmückt, metaphorisch, z.B. der Mythos vom Seelengespann Phdr. 246a ff.) Reden und Seelen. Sein Gesprächspartner Phaidros besitzt mit hoher Sicherheit eine „bunte“ Seele, vgl. Colloud-Streit, Fünf platonische Mythen, 150, und Yunis, Plato: Phaedrus. 
schon inne hat und damit nun noch die Erkenntnis der Zeit, wann geredet und wann inne gehalten werden müsse, verbindet, wenn er ferner für das Kurzreden und die Sprache des Mitleids und der Steigerung, überhaupt für alle Redearten, die er etwa gelernt hat, die rechte Zeit und die Unzeit zu unterscheiden weiß, dann erst ist seine Kunst in schönem und vollkommenem Maße ausgebildet, eher aber nicht.*

Phdr. 271d ff.

Letztendlich kann eine solche wissenschaftlich begründete Rhetorik angesichts der schier unermesslichen Vielfalt der verschiedenen Seelentypen nur auf einen einzigen Adressaten hin ihre volle Wirkung entfalten. ${ }^{23}$ Gegenüber vielen Adressaten muss sie kapitulieren; daher sieht Sokrates die philosophische Rhetorik hauptsächlich im Gespräch zwischen Lehrer und Schüler, wie es exemplarisch im Phaidros vorgeführt wird. ${ }^{24}$

\subsection{Gottgefälligkeit}

Da die wahre Rhetorik an sich die Philosophie voraussetzt (durch das Ideenwissen, die Psychologie und die dialektische Methode), lässt sie sich nur mit grossem (auch zeitlichem) Aufwand erlernen. Den Vorwurf dieses ungünstigen Kosten-Nutzenverhältnisses kontert Sokrates damit, dass er das Ziel der Rhetorik darin sieht, den Göttern und nicht den Menschen zu Gefallen zu sprechen.

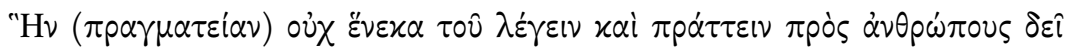

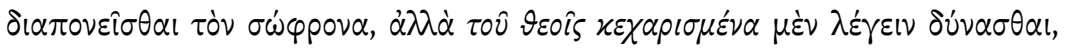

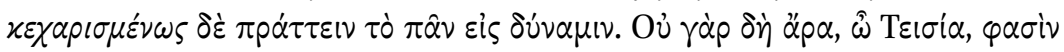

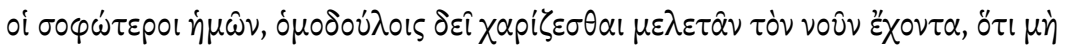

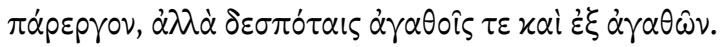

(Sokrates:) Dieser Übung darf sich aber nun der Besonnene nicht um des Redens und Handelns mit Menschen willen unterziehen, sondern um den Göttern Gefälliges reden und in allem nach Vermögen ihnen gefällig handeln zu können. Denn ja nicht darf, o Teisias, - so sagen die, welche weiser als wir sind, - wer Vernunft hat, sich bestreben, seinen Mitknechten sich gefällig zu zeigen, außer in Nebendingen, sondern seinen guten und von Guten kommenden Gebietern.*

Phdr. 273e f.

\footnotetext{
23 Weniger skeptisch gegenüber der Wirkung auf ein grösseres Publikum ist z.B. Yunis, Taming Democracy.

24 Das Scheitern der philosophischen Rhetorik vor der Masse wird z.B. im Gorgias (486a ff. und $521 \mathrm{c} \mathrm{ff.),} \mathrm{im} \mathrm{Theaitetos} \mathrm{(172c} \mathrm{ff.)} \mathrm{und} \mathrm{natürlich} \mathrm{in} \mathrm{der} \mathrm{Apologie} \mathrm{thematisiert.}$
} 
Auch hier ist der Phaidros ein perfekt passendes Beispiel, da er von Beginn an durch das Göttliche geprägt ist und faktisch ein Enkomion auf einen Gott, den Eros, enthält. ${ }^{25}$

\subsection{Distanz zur Schrift, Spiel vs. Ernst, Aussparungsstellen}

Der Phaidros endet mit der bekannten Schriftkritik (Phdr. 274b ff.). Diese ist insbesondere auch eine Kritik an der sophistischen Rhetorik, welche das Buch als Möglichkeit zur Vermittlung ihres Wissens stark propagierte und nutzte. ${ }^{26}$ Als Konsequenzen aus der Schriftkritik ergeben sich für den wahren Redner, dass er

a) den Stellenwert der Schrift für gering hält:

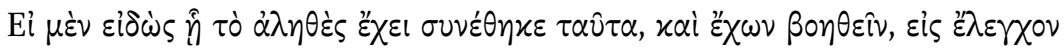

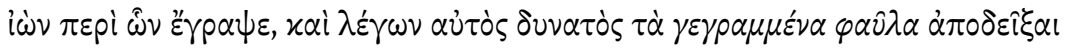
$<$ darf man den Autor als „Philosoph“ bezeichnen $>$.

(Sokrates:) Wenn er dies im Wissen, wie sich dasWahre verhält, verfassthat und ihm helfen kann, indem er sich der kritischen Auseinandersetzung über das, was er geschrieben hat, stellt, und beim Sprechen selbst in der Lage ist, das Geschriebene als minderwertig zu erweisen, < dann darf der betreffende Autor als „Philosoph“ bezeichnet werden>.

Phdr. 278c

b) der Schrift nur Spielerisches anvertraut, während das Ernste (= philosophisch Relevante) nur mündlich tradiert werden kann ( $P h d r .276 \mathrm{~d}$ f., 277e f.).

Platon selbst hat die Problematik der schriftlichen Überlieferung eingesehen und seine Prinzipienlehre in den Dialogen ausdrücklich ausgespart. ${ }^{27}$

\section{Die grosse Rede des Timaios als Beispiel der wahren Rhetorik}

Im Folgenden soll gezeigt werden, dass die Rede des Timaios diese Kriterien weitgehend erfüllt.

\footnotetext{
25 Vgl. Görgemanns, Beiträge zur Interpretation von Platons Nomoi, 63 ff., und Heitsch, „Dialektik und Philosophie in Platons >Phaidros «".

26 O'Sullivan, „Written and Spoken in the First Sophistic“, 119: „the Sophists and the book as their favoured means of communication".

27 Vgl. Szlezák, Platon und die Schriftlichkeit der Philosophie.
} 


\subsection{Kenntnis der Wahrheit (Fachwissen und Ideenwissen)}

Grundsätzlich könnte man die Tatsache, dass im Timaios nicht Sokrates spricht, als Anlass sehen, Platon distanziere sich hier stärker von den Aussagen des Protagonisten. ${ }^{28}$ Allerdings ist Timaios' Rede in vielem derart genuin platonisch, dass eine solche Unterscheidung kaum glaubwürdig erscheint. ${ }^{29}$ Dass sich Platon für Timaios als Redner entschied, hängt wohl eher damit zusammen, dass Sokrates auf dem Gebiet der Kosmologie eben kein ausgewiesener Experte war. Über Timaios wissen wir nur das, was uns Platon zu ihm überliefert: ${ }^{30}$

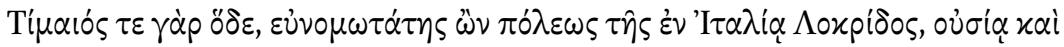

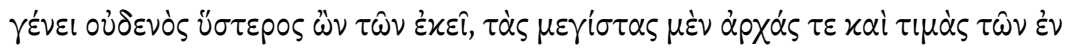

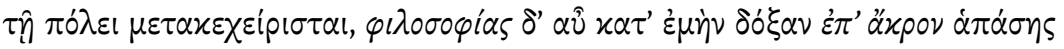
$\dot{\varepsilon} \lambda \dot{\eta} \lambda \cup \theta \varepsilon \nu$.

(Sokrates:) Denn Timaios hier ist aus dem italischen Lokris gebürtig, welches sich der vortrefflichsten Verfassung erfreut, steht keinem von seinen Landsleuten an Vermögen und Herkunft nach und hat dabei einerseits die höchsten Ämter und Ehrenstellen im Staate bekleidet, andererseits in allem, was nur wissenschaftliches Streben heißt, nach meinem Dafürhalten das Höchste erreicht.

Tim. 2oa

Timaios' Voraussetzungen sind also optimal, und er rückt ganz in die Nähe des Philosophenherrschers der Politeia. ${ }^{31}$ Sokrates billigt ihm höchstes philosophisches Wissen zu („wissenschaftliches Streben“ von Susemihl ist viel zu schwach), und gemäss Kritias kann er als Fachexperte für Astronomie gelten.

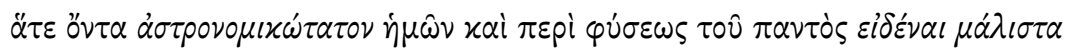
हैp

28 So Bryan, Likeness and Likelihood in the Presocratics and Plato, Lampert und Planeaux, „Who's Who in Plato's Timaeus-Critias and Why", Rowe, Plato and the Art of Philosophical Writing, und Schoos, „Timaeus' Banquet".

29 So Carone, Plato's Cosmology and its Ethical Dimension, Morrow, „Plato's Theory of the Primary Bodies in the Timaeus and the Later Doctrine of Forms“, und Robinson, „The World as Art-Object: Science and the Real in Plato's Timaeus".

30 Vgl. Marg, Timaeus Locrus. De Natura Mundi et Animae, 83: „Nichts nötigt dazu, in der Titelperson von Platons Dialog eine historische Person zu sehen. “̈̈hnlich skeptisch auch Morrow, „Plato's Theory of the Primary Bodies“, und Nails, The People of Plato, s.v. Timaios.

31 So Erler, Die Philosophie der Antike 2/2: Platon, Schofield, „The disappearance of the philosopher king“, und Szlezák, „Über die Art und Weise der Erörterung der Prinzipien im Timaios“. Kritisch Rowe, Philosophical Writing, und Schoos, „Timaeus' Banquet“. 
(Kritias:) weil er sich unter uns am meisten auf die Sternkunde versteht und es sich am meisten zur Aufgabe gemacht hat, über die Natur des Alls zur Erkenntnis zu gelangen.

Tim. 27a

Es spricht also nichts dagegen, dass er die Wahrheit kennt (sofern dies einem Menschen möglich ist), und aus dem Proömium seiner Rede geht klar hervor, dass er auch über Ideenwissen verfügen muss, ganz abgesehen davon, dass er in der Fiktion des Dialogs am Vortag einer Diskussion in der Art der Politeia beigewohnt hat.

Nichtsdestotrotz sind ihm die Grenzen menschlicher Erkenntnis bewusst, wenn er in Bezug auf die gignomena stets nur das „Wahrscheinliche“ (eikos) seiner Darstellung betont und allein dem Gott die Kenntnis der Wahrheit zubilligt.

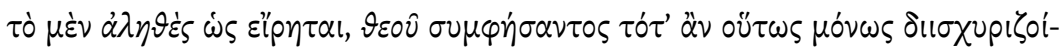

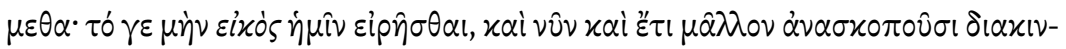

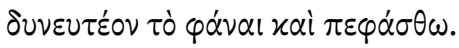

$<$ Das die Seele Betreffende nun> der Wahrheit gemäßangegeben zu haben, das dürften wir wohl nur dann, wenn Gott selbst uns seine Zustimmung dazu gäbe, versichern; daß jedoch wenigstens das Wahrscheinliche hierüber von uns vorgebracht worden, das dürfen wir sowohl schon jetzt, als auch bei noch näherer Betrachtung zu behaupten wagen und wollen es hiermit behauptet haben.

Tim. $72 \mathrm{~d}$

\subsection{Methode zur Erkenntnis der Wahrheit: Dialektik}

Dass Timaios über philosophisches Wissen verfügt, ergibt sich auch daraus, dass er offensichtlich die dialektische Methode kennt und einsetzt, und zwar sowohl das Hypothesisverfahren als auch das Dihairesisverfahren. Auf ersteres weisen

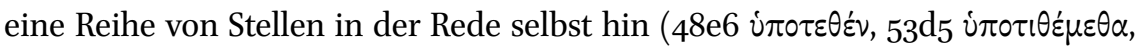

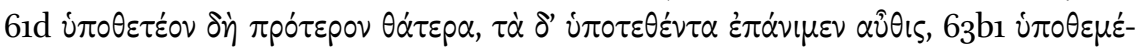
voเs), und Proklos erwähnt dies ausdrücklich in seinem Timaioskommentar.

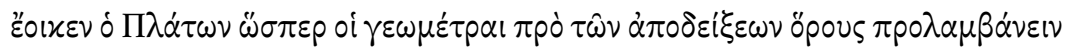
xai i $\pi 0 \theta \varepsilon \dot{\sigma \varepsilon ı \varsigma \varsigma ~}$

Es scheint Platon wie die Mathematiker vor den Beweisen Definitionen und Hypothesen vorwegzunehmen.

Procl., In Tim. I, 236 
Auf letzteres weisen die zahlreichen Kategorisierungen und Differenzierungen in der Rede hin, von denen hier nur die erste erwähnt werden soll.

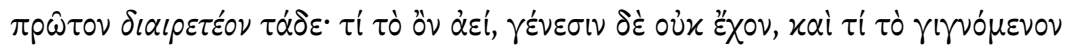

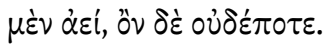

Man muß nun nach meiner Meinung zuerst folgendes unterscheiden und feststellen : wie haben wir uns das immer Seiende, welches kein Werden zuläßt, und wie das immer Werdende zu denken, welches niemals zum Sein gelangt?

Tim. $27 \mathrm{~d}_{5}$

Auch in der Literatur wird in der Regel die Ansicht vertreten, dass Timaios die Dialektik einsetze. ${ }^{32}$

\subsection{Struktur}

4.3.1 Lebewesen (Vollständigkeit, Wohlproportioniertheit)

Timaios unterteilt seine Rede in ein Proömium (Tim. 27c-29d), einen umfangreichen Hauptteil (Tim. 29d-92c) und einen kurzen Epilog (Tim. 92c Criti. 106b). Der Hauptteil selbst gliedertsich in dreigrosse Abschnitteà 17, 20 und 23 Seiten in der Oxfordausgabe, die man ihrerseits in weitere Unterabschnitte zerlegen kann. Als Grundlage für seine Ausführungen verwendet er die Abfolge Kosmogonie-Theogonie-Anthropogonie, die er je durch das Walten des nous, der anankē und beider Kräfte erklärt.

Die Rede deckt ihren Gegenstand offensichtlich vollständig ab:

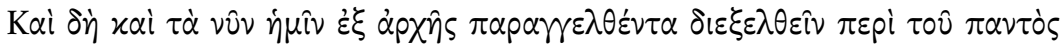

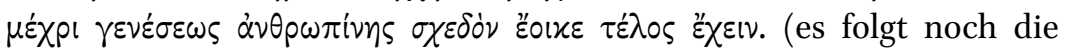
Entstehung der anderen Lebewesen aus dem Menschen)

Und nunmehr scheint denn auch die uns jetzt gesteckte Aufgabe, das Weltall von seinen Anfängen aus bis zur Entstehung der Menschen zu verfolgen, so ziemlich ihr Ziel erreicht zu haben.

Tim. goe

32 Brisson, Le Même et l'Autre dans la structure ontologique du Timée de Platon. Un commentaire systématique du Timée de Platon, 390 mit einer schematischen Darstellung, Runia, „The Language of Excellence in Plato's Timaeus and Later Platonism“, 22: „Platonic dialectic“, Ashbaugh, Plato's Theory of Explanation, 3: „a series of divisions and collections“. Kritisch z.B. Mesch, „Die Bildlichkeit“. 
und ihre Teile sind wie bei einem Lebewesen wohlproportioniert und aufeinander abgestimmt:

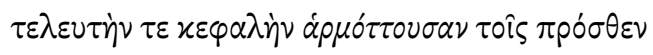

$<$ unserer Dichtung> einen dem Vorhergehenden entsprechenden Schluß hinzuzufügen

Tim. 69b

wie dies auch in der Forschung immer wieder betont wird. ${ }^{33}$

Dass Timaios seine Rede gleichsam dreimal wieder neu beginnen muss, hat zu kontroversen Einschätzungen geführt. ${ }^{34}$ Aus meiner Sicht ist dieses mehrfache neue Einsetzen v.a. auf die Adressatenbezogenheit der Rede zurückzuführen. Er wollte nämlich zunächst das Thema präsentieren, das den Zuhörern am geläufigsten war und auf den meisten Vorkenntnissen aufbaute: Die teleologische Erklärung von der Entstehung des Kosmos als Abbild. Erst nachher ging er auf die komplexeren Einzelheiten der Grundelemente und des menschlichen Körpers ein, welche hohe mathematische und medizinische Anforderungen ans Publikum stellen und bei einer Thematisierung gleich zu Beginn die Gefahr in sich geborgen hätten, dass die Zuhörer überfordert gewesen wären und daher der Rede nicht mehr hätten folgen können und wollen.

\subsection{2 „Logik“ (Argumentation, sinnvolle Gedankenfolge)}

Indem Timaios seine Kosmologie als Kosmogonie gestaltet, folgt er einem narrativen Prinzip und kann so von Beginn an eine „Logik“ in seine Rede einbringen. ${ }^{35}$ Diese besticht zudem durch zahlreiche Aufzählungen, Differenzierungen und

33 Ashbaugh, Plato's Theory of Explanation, 73: „the eikōs logos is constructed in the semblance of a living thing“, Johansen, Plato's Natural Philosophy, 171: „In the Timaeus this idea [von der Rede als Lebewesen] is applied with particular pertinence since the subject matter of Timaeus' speech is itself an animal“.

34 Vgl. e.g. Morgan, Myth and Philosophy from the Presocratics to Plato, 274: „there is no sense that the narrative is an organic whole " und umgekehrt Osborne, „Space, Time, Shape, and Direction: Creative Discourse in the Timaeus “, 193 f.: ,the orderly arrangement of Timaeus' description matches the orderly arrangement of the world itself" (siehe auch die vorangegangene Anmerkung).

35 Die „falsche“ Reihenfolge bei der Schaffung des Weltkörpers und der Weltseele (Tim. 34b f.) kann ebenso auf die Adressatenbezogenheit zurückgeführt werden: Timaios beginnt in voller Absicht mit dem Teil, der für seine Zuhörer aufgrund ihres Vorwissens und durch die Argumentationsstruktur verständlicher ist, und führt erst nachher die mathematisch komplexe Mischung der Weltseele ein. 
Kategorisierungen, Binnenverweise und kurze Zusammenfassungen, und ihre Aussagen werden in der Regel bewiesen.

\subsubsection{Definition}

Timaios definiert jeweils zu Beginn die entscheidenden Begriffe - on und gignomenon (28a), chōra (49a, 51a), dēmiourgos (28a, 29a, 29e), chronos (37d), die vier Grundelemente (51b) - und der Unterbruch nach dem Proömium (29d) ist gerade darauf zurückzuführen, dass er dem Publikum die Gelegenheit geben wollte, zu seinen Grundaussagen und insbesondere zu seinen Definitionen Stellung zu nehmen. Exempli gratia sei hier auf die Definition des Kosmos hingewiesen, der sowohl von der Begrifflichkeit her (ouranos, kosmos, pan) erklärt wird als auch als gignomenon und eikōn seine spezifischen Eigenschaften erhält (28b ff.).

\subsubsection{Länge}

Da die Rede des Timaios kein dialektisches Gespräch unterbricht und verunmöglicht, stellt sie für Sokrates nicht grundsätzlich ein Problem dar. Denn sie ist sowohl inhaltsadäquat, wie dies in der Forschung immer wieder konstatiert wird, ${ }^{36}$ als auch adressatengerecht, da die beiden anderen Zuhörer neben Sokrates einem ungleich längeren und sicher nicht weniger anspruchsvolleren Dialog in Form einer Politeia offenbar ohne Schwierigkeiten folgen konnten, wie aus dem Einleitungsgespräch des Timaios hervorgeht. Zudem ist zu berücksichtigen, dass die Rede sogar kürzer ausgefallen ist, als theoretisch nötig gewesen wäre, da Timaios gewisse Teile weglassen kann:

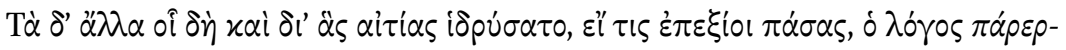

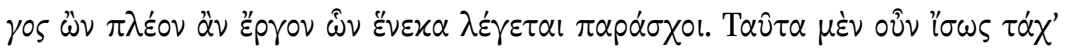

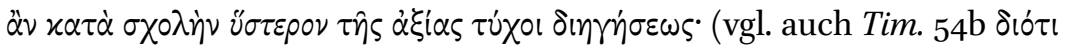

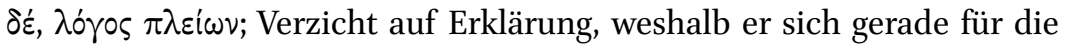
beiden rechtwinkligen Dreiecke entscheidet)

Was aber die übrigen anlangt, so würde, wenn man von allen angeben sollte, wohin und aus welchen Gründen er sie dahin versetzte, diese Auseinandersetzung, die doch nur eine beiläufige wäre, umständlicher sein als die Erörterung selber, welche uns hieraufgeführt hat. Vielleicht wird denn auch dieser Gegenstand späterhin bei größerer Muße eine Darlegung finden, wie er sie verdient.

Tim. $38 \mathrm{~d}$ f.

36 9oes emmetroteros. Vgl.Johansen, Plato's Natural Philosophy, und Mesch, „Die Bildlichkeit“. 


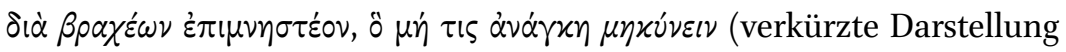
der Entstehung von Frauen und Tieren)

$<$ die Entstehung von Frauen und Tieren> ist nur noch kurz zu erwähnen, es sei denn, daß die Sache hier und da ein Mehreres fordert.

Tim. goe

\subsection{Adressatenbezogenheit}

Wir haben bereits im Zusammenhang mit der Struktur und der Länge gesehen, dass die Rede offensichtlich adressatenbezogen ist.

Auch wenn das Publikum aus drei (statt, wie im Idealfall gefordert, einer) Personen besteht, so verfügen die Zuhörer doch über eine enge Seelenverwandtschaft und repräsentieren eine absolut hochkarätige Gesprächsrunde. So sagt Sokrates selbst über Kritias und Hermokrates: ${ }^{37}$

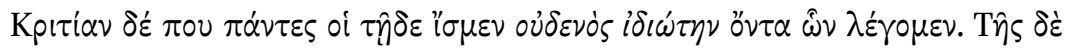

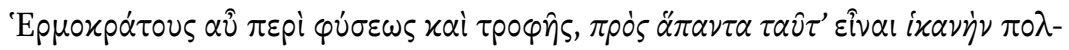

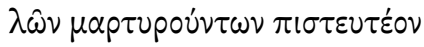

Von dem Kritias aber wissen wir Athener es ja alle, daß ihm nichts von den Dingen, um welche es hier sich handelt, fremd ist, und ebenso darf man es von der Naturanlage wie der Bildung des Hermokrates glauben, daß sie ihnen allen gewachsen sei, da dies von so vielen Seiten bezeugt wird.

Tim. $20 \mathrm{a}$

Man kann wohl zurecht mit Erler sagen: „Als Besonderheit gegenüber anderen Dialogen ist festzuhalten, dass Sokrates' Gesprächspartner im ,Timaios' als besonders qualifiziert, kundig in der Wissenschaft (Tim. 53c) und ihm geradezu gleichrangig vorgestellt werden“, ${ }^{38}$ und man kann ihnen mit Bestimmtheit eine hohe Kompetenz zugestehen, aber sie sind kaum in allen Fällen als eigentliche Philosophenherrscher im Sinn der Politeia zu sehen; ihre unterschiedliche Charakterisierung weist zudem auf eine gewisse Binnendifferenzierung hin.

Die Adressatenbezogenheit kann als gute und plausible Erklärung für die oben erwähnten strukturellen „Defizite“ der Rede (d.h. den zweimaligen Neubeginn) verwendet werden, indem Timaios seine Zuhörer dort abholt, wo sie stehen (nämlich bei der Politeia, d.h. auf einem philosophisch sehr hohen Niveau):

$37 \quad$ Zu Timaios vgl. oben D.1.

38 Erler, Platon, $263 \mathrm{f}$. 


\begin{abstract}
Thema
Notwendige Vorkenntnisse

1) Proömium

Philosophie

2) Weltkörper

basiert auf 1) und minimalen Kenntnissen der Physik (Existenz von vier Grundelementen)

3) Weltseele

Mathematik und Astronomie (Kenntnisse werden vertieft)

4) Elemente Mathematik und Physik (Kenntnisse werden vertieft)

5) Mensch Medizin (Kenntnisse werden vertieft)

Zudem lässt sich durch die Ausrichtung auf das Publikum der zurückhaltende Einsatz von Stilmitteln der sophistischen Rhetorik (so insbesondere der weitgehende Verzicht auf den Parallelismus und das Parison bzw. Isokolon, während umgekehrt z.B. das Polyptoton bei Timaios viel häufiger auftritt) erklären: Sie sind gar nicht notwendig, um überzeugend zu wirken. Ebenso ist darin eine Begründung für die Metapher der Kosmogonie und des Demiurgen zu sehen: Beides ermöglicht nämlich die Darstellung auf der Basis der philosophischen Vorkenntnisse der Zuhörer.
\end{abstract}

\title{
4.5 Gottgefälligkeit
}

Ähnlich wie den Phaidros prägt auch den Timaios das Göttliche ungemein. Er

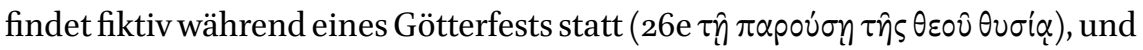
er ist faktisch eine Lobrede auf den Demiurgen und den von ihm geschaffenen göttlichen und von Göttern beherrschten Kosmos. Timaios wendet sich gleich zweimal an die Götter (27c f. s.o. und beim zweiten Proömium in 48d):

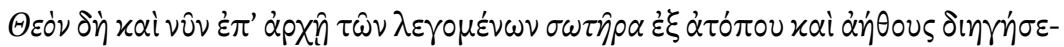

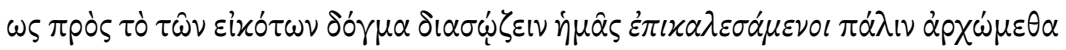
$\lambda \dot{\varepsilon} \gamma \varepsilon \iota$.

Gott also wollen wir auch jetzt bei diesem neuen Beginne unserer Auseinandersetzung anrufen, daß er uns glücklich durch diese fremdartige und ungewöhnliche Darstellungsweise hindurchführen und uns zur wahrscheinlichen Ansicht verhelfen wolle, und dann wirklich von neuem beginnen!

Tim. 48d f.

und insbesondere bittet er nach seiner Rede im Kritias um die wohlwollende Aufnahme durch den Gott:

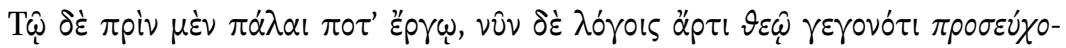

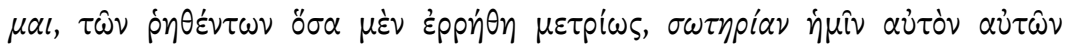




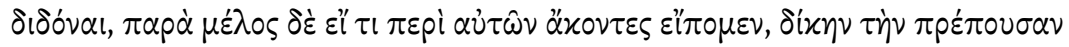

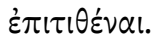

Nun bete ich aber zu dem soeben in meiner Rede entstandenen Gott, uns selbst über das Gesagte, sofern es korrekt gesprochen war, unterstützende Zustimmung zu geben, wenn wir jedoch etwas darüber unabsichtlich falsch gesagt haben, uns die passende Strafe aufzuerlegen.

Criti. 106a f.

Auch in ihrer ethischen Ausrichtung ist die Rede ganz sicher gottgefällig.

\subsection{Distanz zur Schrift}

Als Rede erfüllt Timaios' Darstellung natürlich die Konsequenzen der Schriftkritik per se, und dies kann als ein Grund für die Wahl einer Rede statt eines schriftlichen Traktats angesehen werden.

4.6.1 Spiel vs. Ernst

Darüber hinaus ist die im Phaidros erwähnte Differenzierung zwischen Ernst (der Philosophie) und Spiel (allem anderen) auch Timaios geläufig, wenn er sagt:

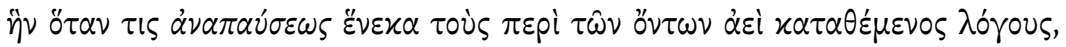

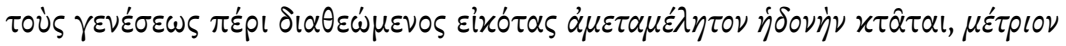

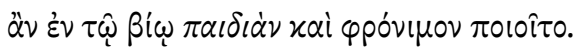

und wenn man einmal zum Zwecke der Erholung die Untersuchungen über das ewig Seiende zur Seite legt und auf die über das Werden, welche nur Wahrscheinlichkeit gewähren, sein Augenmerk richtet und sich so einen Genuß, dem keine Reue folgt, bereitet, so hat man damit für sein Leben eine unterhaltende Beschäftigung gewonnen, wie sie angemessen und verständig ist.

Tim. 59c f.

4.6.2 Aussparungsstellen

Zudem verhindert Platon durch gezielte Aussparungsstellen, dass selbst in der schriftlichen Version der Rede ein Wissen über die Prinzipienlehre dem problematischen Instrument der Schrift anvertraut wird. So verzichtet er auf eine exakte Darstellung des Demiurgen (28c), der archē aller Dinge (48c) und der archē der Grundelemente (53d). ${ }^{39}$

39 Vgl. Szlezák, Platon und die Schriftlichkeit. 


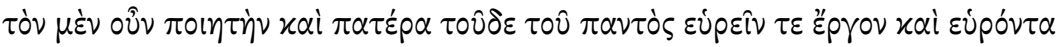

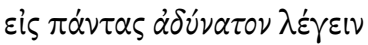

Den Schöpfer und Vater dieses Alls nun zu finden ist freilich schwierig, und wenn man ihn gefunden hat, ist es unmöglich, sich für alle verständlich über ihn auszusprechen.

Tim. 28c

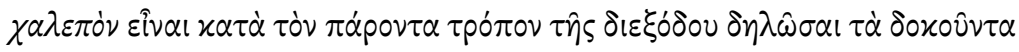

$<$ weil> es zu schwer ist, nach dem ihr zugrunde gelegten Verfahren meine Ansicht hierüber kund zu tun

Tim. 48c

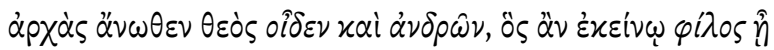

die noch ursprünglicheren Urbestandteile aber kennt nur Gott und von den Menschen etwa der, den er lieb hat

Tim. 53d

\section{7 eikōs logos und eikōs mythos}

In der Einleitung habe ich bereits auf die Relativierung des Wissens hingewiesen, welche Timaios durch die Einführung des eikōs logos vornimmt, indem er eine völlig exakte und widerspruchsfreie Darstellung der gignomena verneint (Tim. 29c). Hier sollen noch ein paar Gedanken zum eikōs logos bzw. mythos sowie zum logos-mythos-Gegensatz folgen, der in der Forschung immer wieder Anlass zu Diskussionen gegeben hat. ${ }^{40}$

Man kann unschwer erkennen, dass eikōs logos im Singular stets ein einzelnes, konkretes, bereits erbrachtes, in der Regel logisch (oder mathematisch) nachvollziehbares Argument bezeichnet (Tim. 3ob7, 53d 5 f., 55 5 , 56a1, 56b4, 9oe8; 57 d6 thematisiert allgemein ein solches Argumentieren); im Plural werden eikotes logoi ausschliesslich in methodologischem Kontext als Argumente verwendet $(29 \mathrm{c} 2,29 \mathrm{c} 8,48 \mathrm{~d} 2)$. Der eikōs mythos steht im Singular für die ganze Darstellung $(29 \mathrm{~d} 2,68 \mathrm{~d} 2)$ oder im Plural generell für jede Art solcher Reden (59c6), die hinwiederum aus eikotes logoi aufgebaut ist.

40 Vgl. u.a. Brisson, „Why is the Timaeus called an eikôs muthos and an eikôs logos?“, Bryan,

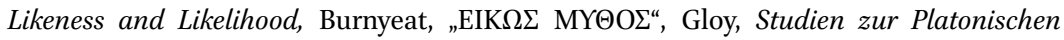
Naturphilosophie im Timaios, und Johansen, Plato's Natural Philosophy. 
Eine Rede als eikōs mythos hebt gleichsam doppelt ihre Defizienz hervor, indem sie einerseits „nur" eikōs ist (d.h. nicht völlig widerspruchsfrei und exakt ist bzw. nur Argumente mit einer solchen Einschränkung enthält, vgl. 29c) und andererseits „nur" ein mythos (d.h. eine nicht verifizierbare Geschichte mit göttlichem Beiwerk). Und gleichzeitig ist vor dem Hintergrund von Kritias' Auffassung, wie sie sowohl im Einleitungsgespräch des Timaios (Tim. 22c, 23b, $26 \mathrm{cff}$.) als auch im Einleitungsgespräch des Kritias (Criti. 106c ff.) vorkommt, der eikōs mythos nur aus historischer oder poetologischer ${ }^{41}$ Sicht dem angeblichen logos unterlegen. Epistemologisch steht der "Mythos" des Timaios Sokrates' philosophischer Erörterung über den idealen Staat offensichtlich näher als die reine narrative Darstellung des Kritias, bei welcher ein Bezug zu den onta überhaupt fehlt - und deren fehlender Wahrheitsgehalt dem Leser ebenso klar ist.

Mythos und logos werden so gesehen tatsächlich austauschbar, wie es in der Forschung teilweise postuliert wird, ${ }^{42}$ aber eben nicht als Begriffe an sich und mithin nicht als Zeichen einer unpräzisen Definition durch Timaios, sondern als Ausdruck der unterschiedlichen Perspektive: Von aussen her, aus der Sicht von (fiktiv Sokrates' und real Platons) Zeitgenossen ist die Darstellung ein Mythos, von innen her jedoch ein Logos beziehungsweise genauer angesichts der von Timaios vertretenen Einschränkungen, die durch das Subjekt des Redners und das Objekt der gignomena gegeben sind, fast ein Logos.

Das von Timaios benutzte eikos stimmt im Übrigen nicht mit dem eikos der sophistischen Rhetorik überein, das sich durch drei Kriterien fassen lässt:

(1) was den Zuhörern wahrscheinlich oder plausibel erscheint (Phdr. 26oa2, 273b1)

(2) was wichtiger ist als die Wahrheit (Phdr. $26 \circ a 3$ f., $272 d 8$ f., $267 a 6$ f.)

(3) was teilweise anstelle der Wahrheit zu verwenden ist ( $P h d r .272 \mathrm{e} 2 \mathrm{f}$.)

(3) gilt sicher nicht - man denke nur an das abschliessende Gebet im Kritias (Criti. 106a f.) -, ebenso (2). Denn Timaios erstrebt an sich die Wahrheit, die aber im Bereich der gignomena zumal für einen Menschen nicht vollumfänglich erreichbar ist. Und auch (1) trifft nicht zu: Timaios erzählt eben nicht das, was seine Zuhörer erwarten, sondern was der - teilweise überraschen-

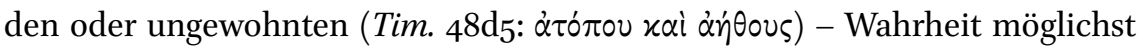
nahe kommt. Zudem lässt Timaios seine Zuhörer nie im Glauben, dass er die uneingeschränkte Wahrheit sagt, sondern kommuniziert gerade an den

41 Vgl. Erler, „Ideal und Geschichte. Die Rahmengespräche des Timaios und Kritias und Aristoteles' Poetik".

42 So e.g. Gloy, Studien zur Platonischen Naturphilosophie im Timaios, und Guthrie, History of Greek Philosophy. 
Stellen, die etwas apodiktisch erscheinen könnten, mit grosser Transparenz die Unvollkommenheit seiner Darstellung.

Die vorangegangenen Ausführungen haben deutlich zum Ausdruck gebracht, dass Timaios in seiner Rede die Kriterien der philosophischen Rhetorik, wie sie hauptsächlich im Phaidros definiert werden, so gut wie möglich einhält. Seine Rede steht in dieser Hinsicht praktisch auf der gleichen Stufe wie die zweite Rede des Sokrates im Phaidros, die ebenfalls als ein Beispiel der wahren Rhetorik angesehen werden kann.

\section{5}

\section{Erklärung der Eigentümlichkeiten}

Damit lassen sich jetzt auch die in der Einleitung aufgeworfenen Fragen oder Auffälligkeiten des Timaios erklären. Das Einleitungsgespräch ist notwendig, um das Kriterium der Adressatenbezogenheit zu erfüllen: Die Rede ist nicht an ein beliebiges Publikum gerichtet, sondern explizit auf Sokrates, Kritias und Hermokrates mit ihren spezifischen Voraussetzungen zugeschnitten. Dessen muss sich umso mehr auch ein potenzieller Leser des Dialogs bewusst sein.

Der Götteranruf ist in der Tat teilweise der Tradition geschuldet (als Verweis auf die mythischen Kosmogonien von Platons Vorgängern, so insbesondere auf Hesiod), doch wird er von Timaios hauptsächlich um des Inhalts willen verwendet, da nur die Götter die Wahrheit der Darstellung verbürgen können. ${ }^{43}$

Das scheinbar unschlüssige Hin- und Herschwanken zwischen mythos und logos lässt sich als absolut geschicktes Verfahren des Timaios interpretieren, seine aus zeitgenössischer Sicht durchaus mythische Rede nicht als wissenschaftlich oder philosophisch minderwertig zu markieren. Auf Kritias' Kategorisierung von Sokrates Staatsutopie als mythos plastheis (Tim. 26e4) antwortet Timaios, indem er seine eigene Kosmogonie als mythos eikōs (Tim. 29d2) bezeichnet und damit geradezu eine neue Gattung definiert.

Die mündliche Darstellungsform basiert auf den Grundlagen der Schriftkritik, die ein situationsbezogenes Gespräch der unveränderlichen Fassung eines Textes vorzieht. Dass die Rede von Platon dann doch als Schrift publiziert worden ist, widerspricht diesen Vorgaben nicht, da sie den Kernbereich der Philosophie, die (ungeschriebene) Prinzipienlehre, fast vollständig ausblendet.

43 Auch im Phaidros kommt ja ein Musenanruf vor - notabene zu Beginn der sonst eher nüchtern-argumentativen ersten Rede (Phdr. 237a). 
Sokrates' Interesse für naturphilosophische Themen im Timaios steht nicht im Gegensatz zum üblichen Bild aus den anderen Dialogen. Denn Timaios vertritt genau die teleologische Variante der Kosmologie, die sich Sokrates auch von Anaxagoras erhofft hätte. ${ }^{44}$ Dazu kommt, dass sie auf Platons Unterscheidung zwischen onta und gignomena basiert und unsere Welt nicht in Konkurrenz zur Ideenwelt erklärt, sondern als Ergänzung mit der notwendigen Relativierung der Erkenntnisse.

Wenn Timaios dazu immer wieder auf den Begriff des eikos zurückkommt, verwendet er zwar ein Schlagwort der sophistischen Rhetorik, aber in einer völlig verschiedenen Funktion und vor allem so transparent, dass die Zuhörer dadurch nicht getäuscht und mutwillig von der Wahrheit weggeführt werden.

Die ungewöhnliche Länge der Rede widerspricht Sokrates' Ansichten im Gorgias und Protagoras nicht, da sie eben nicht ein dialektisches Gespräch unterbricht oder beeinträchtigt. Sie ist stattdessen durch den Inhalt bedingt, und sie entspricht den Aufnahmefähigkeiten ihrer Adressaten.

\section{Vgl.}

$\begin{array}{ll}\text { Allgemein } & \text { Einbettung der Rede in einen } \\ & \text { Dialog, Unterbruch } \\ & \text { Götteranruf } \\ & \text { Mythos und logos } \\ & \text { Mündlichkeit statt Schriftlichkeit } \\ \text { Platon } & \text { Inhalt (naturphilosophische } \\ & \text { Erklärung der Welt) } \\ & \text { Prinzip des eikos } \\ & \text { Länge }\end{array}$

Begründung

Adressatenbezogenheit

Gottgefälligkeit

Aussen- vs. Innensicht

Schriftkritik

Teleologie, onta - gignomena

Epistemologie

Sach- und Adressatenadäquatheit

Soweit sich also Platons Forderungen an eine philosophische Rhetorik aufgrund der teilweise offenen Aussagen im Phaidros überhaupt verifizieren lassen, sind sie in der grossen Rede des Timaios konsequent zur Anwendung gebracht worden. Besonders die Forderung, der Redner müsse die Wahrheit über seinen Gegenstand kennen, das Kriterium der passenden Strukturierung, die Erkenntnisse im Bereich einer wissenschaftlichen Psychologie und die Resultate der Schriftkritik hat Platon in seinem grossen kosmologischen Werk

44 Vgl. Neschke-Hentschke, Le Timée de Platon, contributions à l'histoire de sa réception, $\mathrm{xv}$. Zur teleologischen Welterklärung im Phaidon selbst vgl. Sedley, „Teleology and Myth in the Phaedo". 
folgerichtig in die Praxis umgesetzt und damit die gewaltige Wirkung des Timaios mit grosser Wahrscheinlichkeit überhaupt erst ermöglicht.

\section{Zitierte Literatur}

Ashbaugh, Anne F. Plato's Theory of Explanation. New York: SunY Press, 1988.

Balansard, Anne. Technè dans les Dialogues de Platon. Sankt Augustin: Academia Verlag, 2001.

Brague, Rémi. „The Body of the Speech“. In Platonic Investigations. Herausgegeben von Dominic O'Meara. Washington: Catholic University of America Press, 1985, 53-83.

Brickhouse, Thomas C. und Nicholas D. Smith. Plato's Socrates. New York: Oxford University Press, 1994.

Brisson, Luc. Le Même et l'Autre dans la structure ontologique du Timée de Platon. Un commentaire systématique du Timée de Platon. Zweite Auflage. Sankt Augustin: Academia Verlag, 1994.

Brisson, Luc. „Why is the Timaeus called an eikôs muthos and an eikôs logos?“. In Plato and Myth. Herausgegeben von Catherine Collobert, Pierre Destrée und Francisco Gonzalez. Leiden: Brill, 2012, 369-391.

Bryan, Jenny. Likeness and Likelihood in the Presocratics and Plato. Cambridge: Cambridge University Press, 2012.

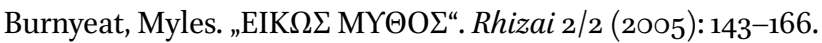

Byrd, Miriam N. „Dialectic and Plato's Method of Hypothesis“. Apeiron 40 (2007): 141-158.

Carone, Gabriela Roxana. Plato's Cosmology and its Ethical Dimension. Cambridge: Cambridge University Press, 2005 .

Colaiaco, James. Socrates against Athens. New York: Routledge, 2001.

Colloud-Streit, Marlis. Fünf platonische Mythen im Verhältnis zu ihren Textumfeldern. Fribourg: Academic Press, 2005.

Erler, Michael. „Ideal und Geschichte. Die Rahmengespräche des Timaios und Kritias und Aristoteles' Poetik". In Interpreting the Timaeus - Critias. Proceedings of the IV Symposium Platonicum. Herausgegeben von Thomas Calvo und Luc Brisson. Sankt Augustin: Academia Verlag, 1997, 83-98.

Erler, Michael. Die Philosophie der Antike 2/2: Platon. Basel: Schwabe Verlag, 2007.

Eucken, Christoph. „Die Doppeldeutigkeit des platonischen Menexenos“. Hyperboreus 9 (2003): 44-55.

Gloy, Karen. Studien zur Platonischen Naturphilosophie im Timaios. Würzburg: Königshausen u. Neumann, 1986.

Görgemanns, Herwig. Beiträge zur Interpretation von Platons Nomoi. München: C. H. Beck, 196o. 
Guthrie, William K. C. A History of Greek Philosophy III-V. Cambridge: Cambridge University Press, 1969-1978.

Hadot, Pierre. „Physique et poésie dans le Timée de Platon“. RThPh 115 (1983): 113-133. Hartmann, Lucius. Die grosse Rede des Timaios - ein Beispiel wahrer Rhetorik. Basel: Schwabe Verlag, 2017 .

Heinimann, Felix. „Eine vorplatonische Theorie der $\tau \varepsilon \dot{\varepsilon} \chi \nu \eta^{\prime}$. Museum Helveticum 18 (1961): 105-130.

Heitsch, Ernst. Platon, Phaidros, Übersetzung und Kommentar. Zweite Auflage. Göttingen: Vandenhoeck \& Ruprecht, 1997.

Heitsch, Ernst. „Dialektik und Philosophie in Platons ,Phaidros““. Hermes 125 (1997): 131-152.

Johansen, Thomas. Plato's Natural Philosophy: A Study of the Timaeus-Critias. Cambridge: Cambridge University Press, 2004.

Lampert, Laurence und Christopher Planeaux. „Who's Who in Plato's Timaeus-Critias and Why". The Review of Metaphysics 52 (1998): 87-125.

Marg, Walter, ed. Timaeus Locrus. De Natura Mundi et Animae. Leiden: Brill, 1972.

Mesch, Walter. „Die Bildlichkeit der platonischen Kosmologie“. In Platon als Mythologe. Herausgegeben von Markus Janka und Christian Schäfer. Darmstadt: WBG Academic, 2002, 194-213.

Morgan, Kathryn A. Myth and Philosophy from the Presocratics to Plato. Cambridge: Cambridge University Press, 2000.

Morrow, Glenn R. „Plato's Theory of the Primary Bodies in the Timaeus and the Later Doctrine of Forms“, Archiv für Geschichte der Philosophie 5o (1968): 12-28.

Nails, Debra. The People of Plato. Indianapolis: Hackett, 2002.

Neschke-Hentschke, Ada, ed. Le Timée de Platon, contributions à l'histoire de sa réception. Louvain: Peeters, 2000.

Nevsky, Alexandre. Voir le monde comme une image. Bern: Peter Lang, 2011.

Osborne, Catherine. „Space, Time, Shape, and Direction: Creative Discourse in the Timaeus". In Form and Argument in Late Plato. Herausgegeben von Christopher Gill. Oxford: Oxford University Press, 1996, 179-211.

O'Sullivan, Neil. „Written and Spoken in the First Sophistic“. In Voice into Text. Herausgegeben von Ian Worthington. Leiden: Brill, 1996, 115-127.

Pernot, Laurent. La rhétorique dans l'antiquité. Paris: Le Livre de Poche, 2000.

Racionero, Quentin. „Logos, myth and probable discourse in Plato's Timaeus“. Elenchos 19 (1998): 29-6o.

Robinson, Thomas M., „The World as Art-Object: Science and the Real in Plato's Timaeus“. Illinois Classical Studies 18 (1993): 99-111.

Rowe, Christopher. Plato and the Art of Philosophical Writing. Cambridge: Cambridge University Press, 2007. 
Runia, David Theunis. „The Language of Excellence in Plato's Timaeus and Later Platonism“. In Platonism in Late Antiquity. Herausgegeben von Stephen Gersh. Notre Dame: University of Notre Dame Press, 1992, 11-37.

Sayre, Kenneth M. Metaphysics and Method in Plato's Statesman. Cambridge: Cambridge University Press, 2006.

Schiappa, Edward. „Did Plato coin Rhêtorikê?“. American Journal of Philology 111 (1990), 457-470.

Schofield, Malcolm. „The disappearance of the philosopher king“. In Boston Area Colloquium in Ancient Philosophy 13. Herausgegeben von John J. Cleary und Gary M. Gurtler, Leiden: Brill, 1999, 213-254.

Schoos, Daniel J. „Timaeus' Banquet“. Ancient Philosophy 19 (1999): 97-107.

Sedley, David. „Teleology and Myth in the Phaedo“. In Boston Area Colloquium in Ancient Philosophy 5. Herausgegeben von John J. Cleary and Daniel C. Shartin, Lanham: University Press of America, 1991, 359-383.

Stenzel, Julius. Studien zur Entwicklung der platonischen Dialektik von Sokrates zu Aristoteles. Zweite Auflage. Leipzig: B. G. Teubner, 1931.

Szlezák, Thomas A. Platon und die Schriftlichkeit der Philosophie. Berlin, 1985.

Szlezák, Thomas A. „Über die Art und Weise der Erörterung der Prinzipien im Timaios“. In Interpreting the Timaeus - Critias, Proceedings of the IV Symposium Platonicum. Herausgegeben von Thomas Calvo und Luc Brisson. Sankt Augustin: Academia Verlag, 1997, 195-203.

Tarán, Leonardo. „The Creation Myth in Plato's Timaeus“. In Essays in Ancient Greek Philosophy. Herausgegeben von John Peter Anton und George Kustas. New York: suNY Press, 1971, 372-407.

Thompson, Wayne N. „The Symposion: a neglected Source for Plato's Ideas on Rhetoric“. In Plato: True and Sophistic Rhetoric. Herausgegeben von Keith Erickson. Amsterdam: Brill 1979, 325-338.

Werner, Daniel. „Rhetoric and philosophy in Plato's Phaedrus“. Greece \& Rome 57 (2010): 21-46.

Yunis, Harvey. Taming Democracy. Ithaca: Cornell University Press, 1996.

Yunis, Harvey, ed. Plato: Phaedrus. Cambridge: Cambridge University Press, 2011. 